\title{
The cross-talk between enterocytes and intraepithelial lymphocytes
}

\author{
Serena Vitale, Stefania Picascia and Carmen Gianfrani
}

\begin{abstract}
The gut mucosa is continuously exposed to food and microbial antigens. Both enterocytes and intraepithelial lymphocytes have a pivotal role in maintaining the integrity of intestinal mucosa, as these cells guarantee a first line of defense against pathogens and toxic molecules. Enterocytes maintain a physical barrier against microbes and directly contribute to the gut homeostasis by sampling the luminal agents through several pattern recognition receptors or presenting antigen to mucosa T cells. Similarly, due to a close physical contact with the intestinal epithelial cells, the intraepithelial lymphocytes represent an important part of the gut lymphoid tissue, contrasting the entry and spread of pathogens. An alteration of the cross-talk between intestinal epithelial cells and intraepithelial lymphocytes might actively contribute to the development of intestinal immune disorders, as occurring in patients with celiac disease. In genetically predisposed individuals, the gluten exposure results in a massive production of interleukin-15, activation of intraepithelial lymphocytes, and modification of small intestinal mucosa architecture and function. We will review the recent studies on the pathophysiology of cross-talk between enterocytes and intraepithelial T cells, and how this interaction is crucial for intestinal integrity and homeostasis.
\end{abstract}

Keywords: Intestinal intraepithelial lymphocytes, Enterocytes, Celiac disease

\section{Intestinal lymphoid tissue}

The gut-associated lymphoid tissue (GALT) is the largest immune compartment of the body, due to the high cellular number and tissue dimension [1]. The GALT is structured in both organized tissues that comprise Peyer's patches (PP) and mesenteric lymph nodes (MLNs), and in diffuse lymphoid tissues, with immunocompetent cells scattered in both the epithelium and lamina propria of the throughout intestinal mucosa [2].

The GALT is daily in contact with a large number of harmful and innocuous antigens. In healthy condition, it efficaciously distinguishes between pathogenic antigens, to which an active immune reaction is needed, and nonpathogenic ones, such as commensal bacteria and food antigens, to which an inflammatory reaction would be deleterious. The GALT unresponsiveness toward innocuous antigens, termed oral tolerance, is a fine balanced and strictly regulated process, and its dysregulation is responsible for development of food allergies and chronic inflammatory disorders [2].

\footnotetext{
* Correspondence: c.gianfrani@ibp.cnr.it

Institute of Protein Biochemistry, CNR, Via Pietro Castellino, 111, 80131 Naples, Italy
}

\section{Intestinal epithelium infiltrating lymphocytes: phenotype and function}

In the epithelium compartment, the GALT is constituted by a heterogeneous population of T lymphocytes (IELs) whose main role is to maintain immune surveillance. Strongly influenced by several luminal factors, as soluble antigens and microbiota, the phenotype and function of IELs markedly change throughout the intestinal tract [3]. 
Whilst in the colon the IELs population is mostly composed of $\mathrm{T}$ cell receptor (TCR) $\alpha \beta+\mathrm{CD} 4+\mathrm{T}$ cells, in the small intestine it is mainly consisted of CD8+ T cells, carrying the $\alpha \beta$ or $\gamma \delta$ TCR. In particular, in the small intestine, $70-80 \%$ of IELs are CD $8 \alpha \beta+$ cells expressing $\mathrm{TCR} \alpha \beta+, 10-15 \%$ are $\mathrm{TCR} \alpha \beta+\mathrm{CD} 4+\mathrm{T}$ cells, and less than $10 \%$ of cells carrying $\mathrm{TCR} \gamma \delta+$ (either CD8+ or double CD8 CD4 negative cells) [3]. The TCR $\alpha \beta+$ CD8 $\alpha \beta+$ IELs may express either the inhibitory or coactivator natural killer receptors (C-type lectins NKRs) [3]. In the absence of biological or physical insults, TCR $\alpha \beta+$ IELs have low cytolytic abilities, express inhibitory CD94/NKG2A and low levels of the activating NKG2D receptor, whilst at the same time intestinal epithelial cells do not express ligands for NKRs. Under physiological conditions, the TCR $\gamma \delta+$ IELs have an antiinflammatory role mediated by the secretion of regulatory cytokines, as IL-10 and TGF- $\beta$. In the presence of inflammatory stimuli, the TCR $\alpha \beta+$ IELs up-regulate the activating NKRs, produce pro-inflammatory cytokines, as TNF- $\alpha$ and IFN- $\gamma$, and trigger the lysis of infected enterocytes by releasing both perforin and granzyme $B$ and thus preventing the entry and spread of pathogens. Indeed, IELs also participate in the epithelial healing by promoting the intestinal epithelial cell (IEC) growth and epithelial layer repair through the keratinocyte growth factor (KGF) release and by removing infected cells [3].

\section{Intestinal epithelial cells: emerging role as non-immune inflammatory cells}

The IECs form a continuous layer that covers the inside part of the gastrointestinal tract surface. In the past, IECs were considered essentially as non-immune cells with a passive role of physical barrier or target of cytotoxic immune cells. Recent studies have underlined the inflammatory role of IECs, in particular as non-professional antigen-presenting cells. Because of the IECs expressing on their surface the major histocompatibility class (MHC) I and II molecules, as well as non-classical MHC molecules (MIC A/B) whose expression increase in the presence of inflammatory stimuli, they are able to process and to present antigens to both IELs and lamina propria $\mathrm{T}$ lymphocytes. Moreover, IECs actively contribute to pathogenic bacteria clearance and to gut homeostasis toward the commensal bacteria, due to the surface expression of pattern recognition receptors (PRRs), as Toll-like receptors (TLRs) and nucleotide-binding oligomerization domains (NODs) [4]. Depending on the intestinal tract sites, the epithelial layer is composed of different types of IECs: enterocytes, goblet, Paneth, and enteroendocrine cells [5]. Each cell subset shows a different morphology and function: the enterocytes have microvilli on their apical surface, whilst the goblet cells contain large vacuoles and secrete mucus; both prevent pathogen invasion.
The enteroendocrine cells, scattered throughout the epithelium, produce hormones involved in cellular tropism, tissue repair, and enterocyte differentiation. Interestingly, recent finding suggested that this cell population has a direct role in the gut response to pathogen and commensal bacteria, acting as innate sensor [6]. Finally, the Paneth cells, mainly placed in the crypts, release defensin peptides against pathogens. Though stem cells in the crypts guarantee a continuous and rapid renewal of the intestinal epithelia, alterations in defensin and mucus production, due to luminal insults, damage the integrity of mucosa barrier and may contribute to intestinal immune-mediated disorders, as inflammatory bowel disease (IBD) and celiac disease (CD) [7].

\section{Enterocytes and T cells in celiac disease}

Adaptive immune response to gluten in the small intestinal mucosa of celiac patients

Celiac disease $(\mathrm{CD})$ is a small intestinal disorder provoked by a dysregulated immune response to wheat gluten and related proteins of barley and rye. This multifactorial disease, caused by both genetic and environmental factors, is characterized by a mild to severe enteropathy and a humoral response to the tissue-transglutaminase autoantigen. HLA class II DQ2 and DQ8 genes are the main risk factors for $\mathrm{CD}[8]$ and present gluten immunogenic peptides to lamina propria $\mathrm{T}$ lymphocytes, as documented by several studies [9]. Following gluten stimulation, a marked proliferation of Th1 and Th0 lymphocytes, and an increased production of inflammatory cytokines, mainly interferon- $\gamma$ (IFN- $\gamma)$, characterize the celiac intestinal mucosa. Up to now, several peptides, derived from gliadins $(\alpha-, \omega-$, and $\gamma$-gliadins $)$ and from glutenins, have been identified to stimulate CD4+ T cells in CD subjects $[9,10]$. Remarkably, gluten-specific CD4+ T cells can be isolated from the small intestinal biopsies of $\mathrm{CD}$ patients and not from non-celiac controls, thus underlining their pathogenic relevance [11].

Despite the pivotal role played by CD4+ T lymphocytes in the pathogenesis of $\mathrm{CD}$, recent evidences suggested the involvement of $\mathrm{CD} 8+\mathrm{T}$ cells in the inflammatory cascade elicited by gluten. The extensive infiltration of CD8+ T lymphocytes in the mucosa of $\mathrm{CD}$ patients, both in the epithelium and lamina propria, represents a histopathological hallmark [12]. Notably, gliadin contains peptides able to bind HLA class I molecules on the surface of antigen-presenting cells [11]. We have found that a short peptide of 10 amino acid length (QLIPCMDVVL), mapping the 123-132 region of $\alpha$-gliadin stimulates CD8+ T lymphocytes in both peripheral blood and intestinal mucosa of HLA A2-positive celiac patients [13]. A further study with organ culture of celiac mucosal explants has demonstrated that this peptide specifically stimulates cytotoxic $\mathrm{CD} 8+\mathrm{T}$ cells resident in the lamina propria and 
induces the apoptosis of enterocytes [14]. Interestingly, initial findings associated the HLA class I $A * 01$ and $B * 08$ genes with susceptibility to celiac disease (CD), though further analysis showed a primary association with alleles encoding for HLA DQ2/8 molecules [15]. By using bioinformatic algorithms for the prediction of peptides with binding motives to surface molecules encoded by $\mathrm{A} * 01$ and $\mathrm{B}^{*} 08$ genes, we found several sequences in all three main gliadin families that bound to either HLA A1 or B8 molecules with high affinity. Selected peptides also recalled IFN- $\gamma$ responses in $A * 01+$ and $B * 08+C D$ patients (Picascia et al. manuscript in preparation). A recent genome-wide association study (GWAS) has highlighted the role of $\mathrm{B} * 08$ gene, in strong linkage disequilibrium with DR3-DQ2.5, as additional susceptibility factor for CD [16]. Altogether, these findings sustain our previous data on HLA class I-restricted T cell responses in CD and strongly encourage further studies to dissect this, still unexplored, pathway in $\mathrm{CD}$ pathogenesis.

\section{Innate immune response to gluten in the small intestinal mucosa of celiac patients and the role of IL-15}

A consistent number of studies have demonstrated that gliadin contains peptides, particular mapping the NH2terminal part of $\alpha$-gliadin, able to stimulate innate immuno-competent cells, or even to exert a direct cellular toxicity [17]. After a brief incubation with gliadin peptide p31-43, Maiuri and co-workers reported that the uninflamed $\mathrm{CD}$ mucosa up-regulated the expression of ICAM-1, CD69, and HLA-DR markers and the density of interleukin-15 (IL-15)-positive cells. Upon a longer stimulation, an increased expression of CD83 on dendritic cells and of CD25 on T cells and macrophages was observed. This peptide also induced intraepithelial migration of CD8+ T cells in treated CD mucosa and an epithelial damage mainly due to enterocyte apoptosis. Moreover, the same authors found that an early activation of the innate cells might favor the capacity of gliadin peptides, particularly p31-43, to stimulate the adaptive $\mathrm{T}$ cell response to immunodominant gliadin epitopes [18]. IL-15 seems to have a key role as mediator of the gliadin-elicited innate activation, as the use of specific neutralizing antibodies inhibited a great part of the above pathways [18].

IL-15 is a pleiotropic pro-inflammatory cytokine, member of the IL-2 family, involved in several mechanisms of both immune and non-immune systems. It is expressed on the surface of mainly monocytes, macrophages, dendritic and epithelial cells in response to inflammatory stimuli. IL-15 has numerous activities, including anti-apoptotic function, stimulation of $\mathrm{T}$ cell proliferation, generation of cytotoxic $\mathrm{T}$ lymphocytes, and regulation of NK cell survival and function, as well as the induction of immunoglobulin synthesis by $\mathrm{B}$ cells.
IL-15 is also an important growth and activator factor for both IELs and IECs. In small intestinal biopsies of healthy subjects, IL-15 protein is almost absent in the lamina propria but expressed, though at low intensity, on villous enterocytes. By contrast, in inflammatory condition, as in active CD mucosa, IL-15 is highly expressed by lamina propria mononuclear cells and by intestinal epithelial cells. The overexpression of IL-15 in the epithelium layer has a key role in TCR-independent expansion and activation of IELs in CD mucosa with villous atrophy [19]. Mention et al. reported that IL-15 was massively expressed at the surface of IECs and it was associated with an enhanced transcription of IFN- $\gamma$ in untreated $\mathrm{CD}$ and in refractory celiac sprue patients (RCS), a pathological condition, characterized by massive intraepithelial infiltration with abnormal phenotype and unresponsive to the gluten-free diet [20]. The increased IL-15 levels on enterocytes selectively promoted the growth and activation of clonal CD103+ CD3- IELs in RCS.

Furthermore, it was demonstrated that in CD, IL-15 induced on the epithelial cells similar effects to those elicited by gliadin, as the TFR and FAS expression in both crypt and villous enterocytes, and the up-regulation of Ki67 expression, a marker of cell proliferation. Overall, these results suggested that gluten-induced IL-15 has a pivotal role in the epithelial layer and lamina propria modifications in CD mucosa [21].

There is a general consensus that the mechanisms involved in the villous atrophy in patients with full-blown $\mathrm{CD}$ include both the intensive epithelium infiltration by $\mathrm{T}$ cells and the increased expression of IL-15, and of non-classical MHC class I molecules, as MICA and MICB, on IECs. MICA and MICB proteins are ligands for the activating NKG2D receptors expressed on the surface of both TCR $\alpha \beta$ and TCR $\gamma \delta$ CD $8+$ T cells. MICs act as cellular stress signals and their recognition by NKG2D receptors induces several immune mechanisms, such as cellular cytotoxicity, proliferation, and cytokine secretion. IECs in the inflamed intestinal mucosa of CD patients show an increased expression of non-classical MHC class I molecules. At the same time, the IELs from CD subjects express high levels of the activating NKG2D and CD94/NKG2C receptors and mediate the killing of enterocytes via NKG2D. Additionally, IL-15, by upregulating the NKG2D receptors, switches on the cytotoxic activity of IEL directed on the neighbor IECs [22]. As previously mentioned, in normal conditions, the cross-talk between IECs and IELs mediated by NKG2D/ MIC might maintain the epithelium in a healthy condition eliminating infected cells. By contrast, in the case of uncontrolled IL- 15 production by IECs induced by gluten, as in celiac intestinal mucosa, intraepithelial CD8+ $\mathrm{T}$ cells are converted into activated killer cells and contribute to epithelial cell destruction and tissue atrophy 
through a TCR-independent NKG2D signaling pathway [23]. A recent study by Setty and co-workers showed that IECs from healthy subjects with a family history of CD expressed higher levels of IL-15 and heat shock proteins (HSPs) than controls and have ultrastructural epithelial alterations [24]. In addition, they found that the cytotoxic IEL from relatives of CD patients expressed higher levels of activating NK receptors than cells from controls, although at lower levels than patients with active CD. By contrast, IEL from subjects with potential CD failed to up-regulate activating NK receptors, thus suggesting that the fine balance between inhibitory and activating molecules expressed by NK cells is variable in the different forms of the $\mathrm{CD}$. These results re-mark that the innate epithelial stress is an important event to convert IELs into active killer cells and to induce villous atrophy.

\section{Conclusions}

It is becoming clearer that epithelial layer is not only a passive physical barrier against luminal microorganisms and toxic molecules but has a more active role in maintaining the intestinal mucosa integrity. Through a number of surface membrane receptors, enterocytes may act as biosensor of microorganisms preventing their mucosal entry. Furthermore, intestinal epithelial cells take mucosal antigens up and act as non-professional antigen-presenting cells, directly activating the $\mathrm{T}$ cell resident both in the epithelium and lamina propria below. Besides the properties mentioned above, the prominent role of enterocytes as non-immune inflammatory cells is emerging in immunemediated disorders. To throughout dissect the cross-talk between intestinal epithelial cells and lymphocytes of both epithelium and lamina propria compartments, it is needful to understand the physiology of intestinal mucosa, as well as the development of intestinal inflammatory cascade occurring in common, and worldwide growing, intestinal disorders, as CD and IBD.

\section{Abbreviations}

CD, celiac disease; GALT, gut-associated lymphoid tissue; GWAS, genome-wide association study; IBD, inflammatory bowel disease; IECs, intestinal epithelial cells; IELs, intraepithelial lymphocytes; IL-15, interleukin-15; KGF, keratinocyte growth factor; MadCAM-1, mucosal vascular cell adhesion molecule-1; MHC, major histocompatibility class; MLNs, mesenteric lymph nodes; NKRs, natural killer receptors; NODs, nucleotide-binding oligomerization domains; PRRs, patter recognition receptors; RCS, refractory celiac sprue patients; TLRs Toll-like receptors

Received: 10 March 2016 Accepted: 19 May 2016

Published online: 01 June 2016

\section{References}

1. Fehervari Z, Kiyono $H$ (2008) The mucosa: at the frontlines of immunity. Trends Immunol 29(11):503-4

2. Agace WW (2008) T-cell recruitment to the intestinal mucosa. Trends Immunol 29(11):514-22

3. Abadie V, Discepolo V, Jabri B (2012) Intraepithelial lymphocytes in celiac disease immunopathology. Semin Immunopathol 34:551-566
4. Danese $\mathrm{S}$ (2008) Nonimmune cells in inflammatory bowel disease: from victim to villain. Trends Immunol 29(11):555-64

5. Laukoetter MG, Nava P, Nusrat A (2008) Role of the intestinal barrier in inflammatory bowel disease. World I Gastroenterol 14:401-407

6. Worthington JJ (2015) The intestinal immunoendocrine axis: novel cross-talk between enteroendocrine cells and the immune system during infection and inflammatory disease. Biochem Soc Trans 43(4):727-733

7. Roda G, Sartini A, Zambon E et al (2010) Intestinal epithelial cells in inflammatory bowel diseases. World I Gastroenterol 16(34):4264-71

8. Margaritte-Jeannin P, Babron MC, Bourgey M et al (2004) HLA-DQ relative risks for coeliac disease in European populations: a study of the European Genetics Cluster on Coeliac Disease. Tissue Antigens 63:562-7

9. Camarca A, Del Mastro A, Gianfrani C (2012) Repertoire of gluten peptides active in celiac disease patients: perspectives for translational therapeutic applications. Endocr Metab Immune Disord Drug Targets 12:207-219

10. Hardy M.Y, Girardin, A, Pizzey C, et al. (2015) Consistency in polyclonal T-cell responses to gluten between children and adults with celiac disease. Gastroenterology 149(6):1541-1552.

11. Gianfrani C, Auricchio S, Troncone R (2005) Adaptive and innate immune responses in celiac disease. Immunol Lett 99(2):141-5

12. Sollid LM (2000) Molecular basis of celiac disease. Annu Rev Immunol 18:53-81

13. Gianfrani C, Troncone R, Mugione P et al (2003) Celiac disease association with CD8+ T cell responses: identification of a novel gliadin-derived HLA-A2-restricted epitope. J Immunol 170(5):2719-26

14. Mazzarella G, Stefanile R, Camarca A et al (2008) Gliadin activates HLA class I-restricted CD8+ T cells in celiac disease intestinal mucosa and induces the enterocyte apoptosis. Gastroenterology 134(4):1017-27

15. Abadie V, Sollid LM, Barreiro LB, Jabri B (2011) Integration of genetic and immunological insights into a model of celiac disease pathogenesis. Annu Rev Immunol 29:493-525

16. Gutierrez-Achury J, Zhernakova A, Pulit SL et al (2015) Fine mapping in the MHC region accounts for $18 \%$ additional genetic risk for celiac disease. Nat Genet 47(6):577-578

17. Maiuri L, Troncone R, Mayer M et al (1996) In vitro activities of A-gliadin-related synthetic peptides: damaging effect on the atrophic coeliac mucosa and activation of mucosal immune response in the treated coeliac mucosa. Scand J Gastroenterol 31(3):247-53

18. Maiuri L, Ciacci C, Ricciardelli I et al (2003) Association between innate response to gliadin and activation of pathogenic $\mathrm{T}$ cells in coeliac disease. Lancet 362(9377):30-7

19. Pagliari D, Cianci R, Frosali S et al (2013) The role of IL-15 in gastrointestinal diseases: a bridge between innate and adaptive immune response. Cytokine Growth Factor Rev 24(5):455-66

20. Mention JJ1, Ben Ahmed M, Bègue B et al (2003) Interleukin 15: a key to disrupted intraepithelial lymphocyte homeostasis and lymphomagenesis in celiac disease. Gastroenterology 3:730-45

21. Maiuri L, Ciacci C, Auricchio S (2000) Interleukin 15 mediates epithelial changes in celiac disease. Gastroenterology 119(4):996-1006

22. Hüe S, Mention JJ, Monteiro RC et al (2004) A direct role for NKG2D/MICA interaction in villous atrophy during celiac disease. Immunity 21(3):367-77

23. Meresse B, Chen Z, Ciszewski C et al (2004) Coordinated induction by IL15 of a TCR-independent NKG2D signaling pathway converts CTL into lymphokine-activated killer cells in celiac disease. Immunity 21(3):357-66

24. Setty M, Discepolo V, Abadie V et al (2015) Distinct and synergistic contributions of epithelial stress and adaptive immunity to functions of intraepithelial killer cells and active celiac disease. Gastroenterology 149(3): 681-91, e10 